\title{
Cognitive screening in brain tumors: short but sensitive enough?
}

\section{Gail A. Robinson ${ }^{1 *}$, Vivien Biggs ${ }^{2}$ and David G. Walker ${ }^{2}$}

${ }^{1}$ Neuropsychology Research Unit, School of Psychology, The University of Queensland, Brisbane, QLD, Australia

${ }_{2}^{2}$ BrizBrain and Spine, The Wesley Hospital, Brisbane, OLD, Australia

\section{Edited by:}

Tamara Ownsworth, Griffith

University, Australia

Reviewed by:

David D. Eisenstat, University of Alberta, Canada

David Shum, Griffith University,

Australia

\section{*Correspondence:}

Gail A. Robinson, Neuropsychology

Research Unit, School of Psychology,

The University of Queensland,

Brisbane, QLD 4072, Australia

e-mail:g.robinson@psy.uq.edu.au

Cognitive deficits in brain tumors are generally thought to be relatively mild and nonspecific, although recent evidence challenges this notion. One possibility is that cognitive screening tools are being used to assess cognitive functions but their sensitivity to detect cognitive impairment may be limited. For improved sensitivity to recognize mild and/or focal cognitive deficits in brain tumors, neuropsychological evaluation tailored to detect specific impairments has been thought crucial. This study investigates the sensitivity of a cognitive screening tool, the Montreal Cognitive Assessment (MoCA), compared to a brief but tailored cognitive assessment (CA) for identifying cognitive deficits in an unselected primary brain tumor sample (i.e., low/high-grade gliomas, meningiomas). Performance is compared on broad measures of impairment: (a) number of patients impaired on the global screening measure or in any cognitive domain; and (b) number of cognitive domains impaired and specific analyses of MoCA-Intact and MoCA-Impaired patients on specific cognitive tests. The MoCA-Impaired group obtained lower naming and word fluency scores than the MoCAIntact group, but otherwise performed comparably on cognitive tests. Overall, based on our results from patients with brain tumor, the MoCA has extremely poor sensitivity for detecting cognitive impairments and a brief but tailored CA is necessary. These findings will be discussed in relation to broader issues for clinical management and planning, as well as specific considerations for neuropsychological assessment of brain tumor patients.

Keywords: neurocognitive deficits, brain tumor, cognitive screening, neuropsychology, brief cognitive assessment, MoCA

\section{INTRODUCTION}

Cognitive function is an independent prognostic factor in the survival of glioma patients $(1,2)$. For brain tumors, cognitive assessment (CA) can inform clinicians of areas to target for neurorehabilitation (3), monitor progress to facilitate decision making about further intervention (4), and if there has been a decline in cognitive function, address the question of whether the tumor has recurred or progressed (3). In addition, a CA is able to address the question of whether subtle alterations in cognitive function are significant or not, particularly when monitoring slow-growing low-grade gliomas (4). Assessment of cognitive status can be undertaken with a brief cognitive screen or by a longer formal neuropsychological evaluation. Cognitive screening is typically used in acute states, at bedside, hence the focus of our study is to identify whether a brief CA can be tolerated and completed in a relatively acute state (post-surgery but $<3$ months) and, if so, whether this yields better results in terms of detecting cognitive deficits.

Cognitive screening tools are popular but their sensitivity to cognitive impairment in general, and specifically for brain tumor patients, has been questioned (4). One reason may be that brain tumor-associated cognitive deficits have been thought to be relatively mild and non-specific (5), although this has recently been challenged (6). It is unsurprising that severity and specificity of cognitive deficits in brain tumor patients has been debated as prevalence rates vary from 29 to $91 \%$. This variability may depend on several factors including time of assessment (pre- or postsurgery), tumor grade, treatments (radiation, chemotherapy), and lesion location (7). However, the main reason for this variability may be the method used to assess cognitive functions. For example, in one study, few patients with low-grade gliomas showed cognitive deterioration when screened with the mini-mental state examination (MMSE) (8), irrespective of radiation treatment (9). By contrast, Tucha and colleagues (10) investigated cognitive function with neuropsychological tests and reported that $91 \%$ of patients with frontal or temporal tumors were impaired in at least one cognitive domain. In this study, we aimed to investigate the most effective and efficient method of detection of cognitive impairments in the acute period following tumor resection by directly comparing a cognitive screening tool with a brief but domain-specific CA.

Cognitive screening tools have the advantage of brevity and simplicity of administration. The main question, however, is whether these tools are sensitive to detect abnormalities. In the last decade, the Montreal Cognitive Assessment (MoCA) (11) screening tool has been increasingly favored over the MMSE as it has been shown to have greater sensitivity for detecting cognitive dysfunction. This has been shown in patients with brain tumors (12) and brain metastases (13), as well as in other neurological conditions including stroke (14), sub-arachnoid hemorrhages (SAHs) (15), and silent cerebral infarcts (16). Bernstein et al. investigated the psychometric properties of the MoCA in three diverse brain 
pathologies and concluded that it was reliable in detecting cognitive dysfunction as well as having the benefit of not fatiguing the patient (17). However, regardless of which cognitive screening tool has the greatest sensitivity, the original purpose of these tools was to detect global or generalized decline rather than domain-specific cognitive deficits. Indeed, the need for domain-specific cognitive tests for the brain tumor population was recently highlighted by a study of glioma patients (6). In this study, a range of specific visuospatial deficits were identified in right parieto-temporal gliomas that were not present in patients with prefrontal tumors Thus, it remains uncertain whether cognitive screening tools are sensitive to identify mild and/or focal deficits in brain tumors $(4,12)$.

Neuropsychological evaluations are held to be the "gold standard" for assessment of cognitive functions in focal neurological disorders like stroke $(15,18)$. However, evaluations differ in test composition and can range from long and comprehensive, with a fixed test battery, to brief and flexible, with tests chosen to assess specific cognitive domains (19). One advantage of neuropsychological evaluation is the freedom to include tests that tap specific cognitive functions, depending on tumor location and presenting symptoms (4). On the other hand, the main criticism is the length of assessment that can range from brief ( $1-2 \mathrm{~h})$ to lengthy $(8 \mathrm{~h})$. Length is a particular issue in brain tumor patients as physical and mental fatigue has specifically been identified as a concern (12, 20). In fact, Olsen and colleagues (12) found a selection bias in which patients were willing to complete a 4-h neuropsychological assessment. In particular, they identified that those who completed both the 4 -h assessment and cognitive screening tests, tended to be younger with a higher level of education, they obtained a higher MoCA score and were on lower doses of medications. Thus, Olsen and colleagues, like Papagno et al. (4), concluded that a brief and well-tolerated CA is desirable, when diagnostic accuracy can be maintained.

Neuropsychological evaluation has been compared to cognitive screening tools. As noted above, Olsen and colleagues (12) compared neuropsychological assessment to both the MMSE and the MoCA. The MoCA showed greater sensitivity to cognitive dysfunction than the MMSE; however, the main conclusion was that inclusion of a 4-h neuropsychological assessment was a significant deterrent for participation. The MMSE and MoCA, compared to neuropsychological assessment and return to work status, have been investigated in patients following aneurysmal SAH (15). In their study, $42 \%$ of patients were impaired on the MoCA, compared to none on the MMSE, and the MoCA correlated with domain-specific cognitive tests while the MMSE showed no association with specific tests. In addition, two MoCA items were associated with return to work. The MoCA was concluded to be more sensitive than the MMSE in SAH; however, it was not clear that the MoCA had sufficient sensitivity when compared to the neuropsychological assessment (15). Recently, a large retrospective study of acute stroke has unequivocally demonstrated that the MoCA underestimated cognitive impairment, compared to a brief 1-2 h neuropsychological assessment (18).

The current study compared the MoCA cognitive screening tool with a brief $1-1.5 \mathrm{~h}$ neuropsychological evaluation in primary brain tumors. The neuropsychological evaluation comprised a CA and mood and behavioral assessments as this is thought important to fully characterize level of function and inform care plans (7). The aim was to ascertain whether the MoCA is sufficiently sensitive to detect cognitive impairment at an acute, post-resection time point or whether a brief but domain-specific CA is necessary.

\section{MATERIALS AND METHODS \\ PATIENTS}

Thirty-six patients with primary brain tumors (low- or high-grade gliomas, meningiomas) were recruited by the Brain Tumor Nurse Practitioner (VB) from BrizBrain and Spine, The Wesley Hospital, Brisbane, QLD, Australia. Ethical approval for the study was granted by the UnitingCare and The University of Queensland Human Research Ethics Committees. Informed and written consent was obtained from all patients. Inclusion criteria were (1) confirmation of brain tumor ascertained by MRI and (2) all patients underwent surgical resection prior to the investigation of cognitive functions. The cognitive screening tool was administered before the CA, which was completed in one testing session. The third (3) inclusion criterion was that the cognitive screening tool and CA were completed within the same week to minimize effects due to timing of cognitive screening or assessment. Thus, due to the latter, only 23 patients aged 18-69 years old were included. The mean time between surgical resection and neuropsychological evaluation was 2.1 months $(\mathrm{SD}=3.1$; see Table 1 for patient characteristics). We note that 2.1 months is sufficient time to allow for findings to be useful for neurorehabilitation (if available), planning for management of deficits for the patient and family/carers, and to address any questions related to returning to community roles at home or work.

\section{COGNITIVE SCREENING}

The MoCA (11) was used as the screening tool. Although it was developed as a brief measure of global cognitive function, it contains items that measure these cognitive domains: visuospatial/executive function; naming; memory; language; abstraction; and attention. Specifically, the MoCA is scored out of 30 points comprising these items: brief trail making, cube copy, and clock drawing (visuospatial/executive domain $=5 \mathrm{pts}$ ); animals to name (naming domain $=3 \mathrm{pts}$ ); five words to recall (memory domain $=5$ pts); three brief attention tasks (attention domain $=6$ pts); sentence repetition and word fluency (language domain $=3$ pts); similarities (abstraction domain $=2 \mathrm{pts}$ ); time/place questions (orientation domain $=6 \mathrm{pts}$ ). A normal score is 26 or above.

\section{NEUROPSYCHOLOGICAL EVALUATION Cognitive assessment}

A brief but tailored CA was administered that was completed in 1$1.5 \mathrm{~h}$, depending on individual patient's level of fatigue and ability. The CA was devised based on neuropsychological assessment principles and assessment of standard cognitive domains, detailed in Cipolotti and Warrington (21). The cognitive tests were specifically chosen based on Robinson's recent lesion studies of brain tumor and stroke patients with focal frontal and non-frontal lesions [e.g., Ref. (22, 23)]. A similar approach was adopted by Papagno and colleagues (4) in their recent study of low-grade gliomas. Thus, estimated pre-morbid level of intelligence was ascertained in a 
Table 1 | MoCA, demographic, and behavioral scores (mean \pm SD): all patients and MoCA sub-groups (MoCA-Intact and MoCA-Impaired)

\begin{tabular}{|c|c|c|c|}
\hline & All $(N=23)$ & $\begin{array}{l}\text { MoCA-Intact } \\
(N=16)\end{array}$ & $\begin{array}{l}\text { MoCA-Impaired } \\
(N=7)\end{array}$ \\
\hline MoCA score $(/ 30)$ & $26.52 \pm 2.11$ & $27.63 \pm 1.15$ & $24.00 \pm 1.53^{* * *}$ \\
\hline Age $(M \pm S D)$ & $48.39 \pm 14.61$ & $46.94 \pm 15.81$ & $51.71 \pm 11.79$ \\
\hline Gender (M:F) & $16: 7$ & $12: 4$ & $4: 3$ \\
\hline Education & $13.90 \pm 2.98$ & $14.33 \pm 2.94$ & $12.83 \pm 3.06$ \\
\hline $\begin{array}{l}\text { Pre-morbid estimated } \\
\text { intelligence (NART IQ) }\end{array}$ & $104.13 \pm 10.35$ & $103.56 \pm 10.24$ & $105.43 \pm 11.30$ \\
\hline $\begin{array}{l}\text { Chronicity (months } \\
\text { post-surgery) }\end{array}$ & $2.07 \pm 3.11$ & $2.54 \pm 3.48$ & $0.67 \pm 0.41$ \\
\hline \multicolumn{4}{|l|}{ Tumor type } \\
\hline Meningioma & 4 & 3 & 1 \\
\hline Oligodendroglioma (II) & 5 & 4 & 1 \\
\hline Astrocytoma (II) & 4 & 2 & 2 \\
\hline Oligodendroglioma (III) & 1 & 1 & 0 \\
\hline Astrocytoma (III) & 1 & 1 & 0 \\
\hline $\begin{array}{l}\text { Glioblastoma } \\
\text { multiforme (IV) }\end{array}$ & 8 & 5 & 3 \\
\hline Tumor location (L/R) & $12 / 11$ & $8 / 8$ & $4 / 3$ \\
\hline Frontal (L/R) & $7 / 3$ & $4 / 2$ & $3 / 1$ \\
\hline Temporal (L/R) & $2 / 3$ & $1 / 2$ & $1 / 1$ \\
\hline Parietal (L/R) & $0 / 2$ & $0 / 2$ & $0 / 0$ \\
\hline$>1$ lobe (L/R) & $2 / 3$ & $2 / 2$ & $0 / 1$ \\
\hline HADS anxiety (/21) & $5.94 \pm 3.86$ & $5.83 \pm 4.17$ & $6.20 \pm 3.43$ \\
\hline HADS depression (/21) & $3.88 \pm 2.62$ & $3.08 \pm 2.43$ & $5.80 \pm 2.17^{*}$ \\
\hline $\begin{array}{l}\text { Apathy Evaluation } \\
\text { Scale (/72) }\end{array}$ & $49.20 \pm 15.41$ & $50.86 \pm 16.17$ & $45.33 \pm 14.05$ \\
\hline
\end{tabular}

NART, National Adult Reading Test; L, left; R, right; HADS, Hospital Anxiety and Depression Scale. ${ }^{* *} p<0.001,{ }^{*} p<0.05$, MoCA-Intact compared to MoCA-Impaired patients.

standard manner by administering the National Adult Reading Test (NART) (24). To ascertain current level of cognitive function, the CA comprised standard published neuropsychological tests that focused on the following domain-specific areas of cognition: (1) Abstract reasoning: non-verbal - Raven's advanced progressive matrices (25), verbal - Proverb Interpretation Test $(26,27)$; (2) attention - Digit Span subtest from the Wechsler Adult Intelligence Scale-III (28), Elevator Counting with Distraction from the Test of Everyday Attention (29); (3) verbal and visual memory - Recognition Memory Tests, Words, and Topography (30, 31); (4) visual perception - Incomplete Letters Test from the Visual Object and Space Perception Battery (32); (5) language - Graded Naming Test (33), Word Comprehension - Synonyms Test (34); and (6) executive functions - phonemic word fluency (35), Hayling Sentence Completion Test (36).

\section{Mood and behavior assessment}

As part of the neuropsychological evaluation, level of self-reported anxiety, depression, and apathy were assessed using the Hospital Anxiety and Depression Scale (HADS) (37) and the Apathy Evaluation Scale (AES) (38). A score on the HADS of 7 or below is in the normal range with a score at or above 11 indicating significant levels of anxiety or depression. The AES results in scores between 18 and 72, with higher scores indicating increased apathy and a score of 41 suggested as the cut-off.

\section{Analyses}

The MoCA and domain-specific cognitive tests were administered and scored in the standard and published manner. Patients were classified as cognitively intact on the MoCA if they obtained a score of $\geq 26$ or impaired if they scored $<26$ (11). For each individual cognitive test, patients were classified as cognitively impaired if they scored $<5$ th percentile (i.e., $5 \%$ cut-off), with an intact performance $\geq 5 \%$ cut-off [for similar methodology, see Ref. (18, 39)]. For the Proverb Interpretation Test of verbal abstraction, an impaired performance was a score of $<5 / 8$ [for scoring details, see Ref. (24)].

Performance was analyzed in several ways. First, we calculated a broad measure of impairment for both the MoCA and the CA. For the MoCA, the number of patients impaired is reported. For the CA, we calculated the number of patients impaired on any test and also the number of cognitive domains each patient was impaired in (i.e., 0-6 cognitive domains). Second, based on the method adopted by Chan and colleagues for stroke patients (18), we conducted two specific analyses: (1) MoCA-Intact patients were investigated for impairment in each cognitive domain assessed by the CA; and (2) Patients who scored the maximum points in each of the MoCA-specified cognitive domains, irrespective of the overall MoCA score, were analyzed in terms of discrepancy between this and performance on the domain relevant CA test. We also analyzed whether the MoCA-Impaired patients were impaired in at least one cognitive domain.

\section{RESULTS}

For the first broad measure, we found that 30.4\% (7/23) of our patients were impaired on the MoCA as they scored $<26$. A summary of the MoCA, demographic, and mood and behavior scores for the whole group, and the MoCA-Intact and MoCA-Impaired sub-groups, are contained in Table 1. As expected, the MoCA score for the impaired group was significantly lower than the intact group, $t(21)=6.31, p<0.001$. Apart from slightly higher selfreported symptoms of depression by the MoCA-Impaired group compared to the MoCA-Intact group, $t(15)=2.16, p<0.05$, the two groups were well matched for age, gender, education, pre-morbid intelligence, and chronicity (time since surgery; all $p>0.05)$. Similarly, there was no difference between these two groups in self-reported anxiety or apathy. With regard to symptoms of depression, we note that the mean of both groups is in the "normal" range and not indicative of clinical or subclinical depression. If we examine individual scores, one patient in each group (MoCA-Intact and -Impaired) was in the abnormal range. For anxiety, abnormal scores were obtained by three patients in each group (MoCA-Intact and -Impaired). Finally, both groups reported mildly elevated levels of apathy with a number of patients in both groups above the suggested cutoff (11 in the MoCA-Intact and 4 in the MoCA-Impaired group), which may reflect the acute post-resection stage of assessment. 
Table 2 | Domain-specific Cognitive Test Scores (mean \pm SD): all participants, MoCA sub-groups (MoCA-Intact and MoCA-Impaired), and comparison statistic between MoCA sub-groups.

\begin{tabular}{|c|c|c|c|c|}
\hline Cognitive domain/test & All $(N=23)$ & MoCA-Intact $(N=16)$ & MoCA-Impaired $(N=7)$ & $p$ Value \\
\hline \multicolumn{5}{|l|}{ Abstract reasoning } \\
\hline Advanced progressive matrices (/12) & $7.09 \pm 2.15$ & $7.31 \pm 2.27$ & $6.57 \pm 1.90$ & $p=0.460$ \\
\hline \multicolumn{5}{|l|}{ Memory } \\
\hline RMT words (/50) & $45.91 \pm 4.51$ & $46.19 \pm 4.52$ & $45.29 \pm 4.79$ & $p=0.670$ \\
\hline Digit span total & $17.00 \pm 5.14$ & $17.00 \pm 4.45$ & $17.00 \pm 6.99$ & $p=1.00$ \\
\hline Elevator counting + distraction (/10) & $5.88 \pm 3.38$ & $6.20 \pm 3.33$ & $5.33 \pm 3.72$ & $p=0.095$ \\
\hline \multicolumn{5}{|l|}{ Language } \\
\hline Graded Naming Test (/30) & $18.22 \pm 3.46$ & $19.31 \pm 2.82$ & $15.71 \pm 3.68 *$ & $p=0.018$ \\
\hline Synonyms $(/ 50)$ & $39.85 \pm 3.66$ & $40.36 \pm 3.75$ & $38.67 \pm 3.45$ & $p=0.358$ \\
\hline Hayling Test Overall Scaled Score $(1-10,6=$ Average $)$ & $3.52 \pm 2.47$ & $3.63 \pm 2.36$ & $3.29 \pm 2.87$ & $p=0.769$ \\
\hline
\end{tabular}

Bold represents a significant finding. ${ }^{*} p<0.05$.

For the CA broad measure, 69.6\% (16/23) of the patients were impaired on at least one domain-specific cognitive test. The means and SDs for the whole group, and the MoCA-Intact and MoCA-Impaired sub-groups, are reported in Table 2. Overall, there was no difference between sub-groups in performance on 9 of the 11 cognitive tests (i.e., $p>0.05$ ), which supports specific patterns of cognitive deficits rather than a generally lower performance of the MoCA-Impaired patients. By contrast, the MoCAImpaired group performed significantly poorer on the Graded Naming Test of language, $t(21)=2.567, p<0.05$, and the phonemic word fluency test that is sensitive to executive dysfunction, $t(21)=2.363, p<0.05$. The number of cognitive domains each patient was impaired in was as follows: $4 / 16$ impaired in one domain; 4/16 impaired in two domains; 6/16 impaired in three domains; and 2/16 impaired in four domains. Thus, $75.0 \%$ of the impaired patients were impaired on tests in at least two cognitive domains.

For the specific measures based on Chan et al. (18), first we investigated the 16 MoCA-Intact patients for impairment in each cognitive domain assessed by the CA. Of these patients, $56.3 \%$ were impaired in at least one of the six cognitive domains. The percentage of MoCA-Intact patients impaired on domain-specific cognitive tests is shown in Figure 1. The main cognitive domains impaired for MoCA-Intact patients were abilities related to higher level executive functions, including abstract reasoning, followed by attention and memory. By contrast, language was only impaired in $<10 \%$ and no patient was impaired on the test of visual perception.

For the second specific measure, we examined patients who scored the maximum points in each of the MoCA-specified cognitive domains, irrespective of the overall MoCA score. Based on Chan et al. (18), we analyzed the discrepancy between this and

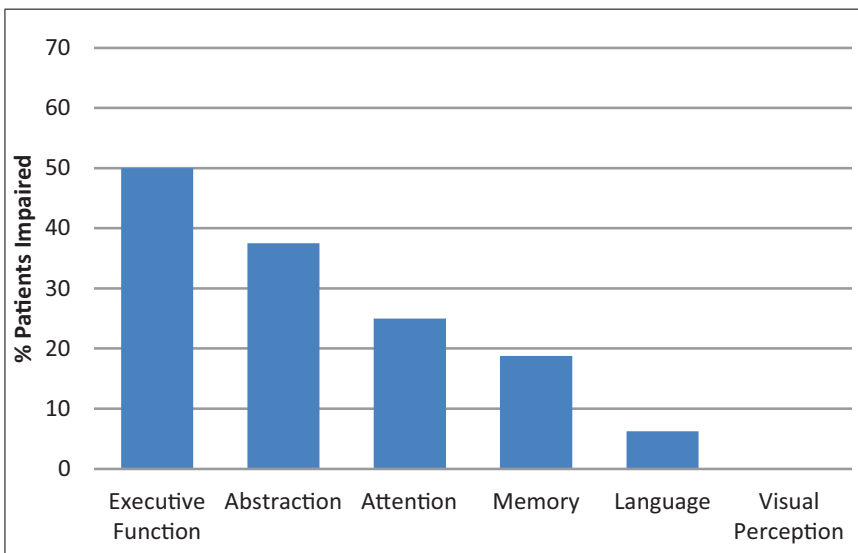

FIGURE 1 | Cognitive assessment: MoCA-Intact participants impaired in domain-specific cognitive tests.

performance on the domain relevant CA test. The number of patients who scored full marks on each MoCA-specified domain, were impaired on the relevant $\mathrm{CA}$ test and the negative predictive values are reported in Table 3.

For the MoCA-Impaired patients, $100 \%$ were impaired in at least one cognitive domain on the CA. Thus, when a patient obtains an impaired score on the MoCA this fully predicts significant impairment in at least one domain on CA. By contrast, the implications for cognitive function is less certain when a "normal" MoCA score is obtained as the MoCA showed very poor negative predictive value (0.44). Further, sensitivity for detecting cognitive impairment is extremely poor (0.44) in our primary brain tumor sample. 
Table 3 | Cognitive assessment performance and negative predictive value for MoCA-specified domains.

\begin{tabular}{lccc}
\hline $\begin{array}{l}\text { MoCA-specified } \\
\text { domain }\end{array}$ & $\begin{array}{l}\text { No. patients } \\
\text { scoring full } \\
\text { marks on MoCA }\end{array}$ & $\begin{array}{l}\text { No. patients (\%) } \\
\text { impaired on CA }\end{array}$ & $\begin{array}{l}\text { Negative } \\
\text { predictive } \\
\text { value (NPV) }\end{array}$ \\
\hline Visuospatial/executive & 13 & $5(38 \%)$ & 0.62 \\
Naming & 21 & $3(14 \%)$ & 0.86 \\
Memory & 4 & $1(25 \%)$ & 0.75 \\
Attention & 13 & $4(31 \%)$ & 0.69 \\
Language & 7 & $3(43 \%)$ & 0.57 \\
Abstraction & 22 & $9(41 \%)$ & 0.59 \\
\hline
\end{tabular}

\section{DISCUSSION}

In our unselected primary brain tumor sample, only $30.4 \%$ were impaired on the MoCA cognitive screening tool. By contrast, for the CA, $69.6 \%$ of patients were impaired on at least one domainspecific cognitive test and, of these, $75 \%$ were impaired in at least two cognitive domains. If we examine the MoCA-Intact patients, more than half $(56.3 \%)$ were impaired in at least one of the six cognitive domains. Specifically, 50\% of the MoCA-Intact patients were impaired on tests of executive function, including abstraction, and a quarter of these patients were impaired in the domains of attention and memory. The level of sensitivity of 0.44 for the MoCA in our patients was far lower than for other neurological disorders. For example, the sensitivity of the MoCA in an acute stroke population was 0.82 (18) and, notably, assessments were completed at comparable times post-stroke or tumor resection. However, we note that the MoCA has been found useful in patients with brain metastases (13) and it is reported to be adequate for the detection of mild cognitive impairment in neurodegenerative disorders such as Alzheimer's and Parkinson's disease [e.g., Ref. (40)]. Nevertheless, the sensitivity of 0.44 of the MoCA for our primary brain tumor population is extremely poor.

In light of this low detection rate of cognitive abnormalities, it is noteworthy that the mean MoCA score of 26.5 for our tumor patients is relatively high and indicative of mild global cognitive impairment. This was also the case for our mood and behavioral measures of anxiety, depression, and apathy. More specifically, the "MoCA-Intact" group obtained a score almost identical to the normal controls reported by Nasreddine et al. (11) while the mean score of 24 for the "MoCA-Impaired" group falls toward the top of the "mild cognitive impairment" group. The overall "mild" level of impairment on the MoCA in our sample differs from the lower MoCA mean score of 22 in patients with brain metastases (13). In fact, Olsen et al. suggested that the MoCA score may be helpful in this population as patients with low MoCA scores may be less likely to benefit from palliative whole-brain radiotherapy while patients with high MoCA scores may tolerate more intensive interventions (13). Thus, for prognostic and treatment purposes in brain metastases, the MoCA may be useful. However, our results at a global level support the notion that primary brain tumor-associated cognitive deficits are indeed mild and/or focal and are hard to detect using global screening tools like the MoCA.
For the $69.6 \%$ of patients impaired in at least one cognitive domain on the CA, executive functions and abstract reasoning were the most common domains impaired by far. In fact, $87.5 \%$ of patients were impaired in these two domains and the remaining two patients presented with a selective nominal aphasia. This is followed by attention ( $43.8 \%$ impaired) and memory $(37.5 \%$ impaired). These cognitive domains being the most often impaired is consistent with the findings of Tucha et al. (10) for frontal and temporal tumor patients. Interestingly, of the two executive function tests, phonemic word fluency and the Hayling Test, 52.2\% of all patients were impaired on just one test, the Hayling Test, which suggests that test choice is critical. With regard to memory, the MoCA does not assess visual memory and $21.7 \%$ of our patients were impaired on our specific visual memory test. By contrast, the intact performance of all our patients on our test of visual perception does not reflect the finding of Shallice and colleagues (6) of visuospatial deficits in right posterior tumor patients. There are two possibilities for the apparent disparity. One, our specific test of visual perception is not sensitive to mild deficits. Two, our seven patients with right posterior tumors are remarkably intact. Upon examination of individual patients, one right temporal MoCA-Impaired and three right posterior MoCAIntact patients lost points on the MoCA-specified visuospatial items. In addition, one of the MoCA-Intact patients presented with a highly selective apperceptive amusia in the context of an otherwise intact cognitive profile (41). This latter case, in addition to the two patients with a selective nominal aphasia, highlight the potential for any cognitive deficit to be specific and focal in brain tumor patients, thus, necessitating freedom in test choice based on symptoms and/or tumor location.

Notably, patients who performed well on MoCA-specified domains were not always intact on the specific cognitive test, similar to Chan et al.'s findings in acute stroke patients. This is particularly so for the abstraction and executive/visuospatial MoCA-specified domains that are assessed by one item each, both clearly insensitive to our patients' deficits. By contrast, the two MoCA-specified domains that most closely resembled the CA impairments were language and memory. In terms of language, only $30.4 \%$ of patients scored full marks on the MoCA-specified items that comprised a sentence repetition item ( $>10$ words in length) and a phonemic word fluency task. Of these two items, phonemic word fluency was one of two standard cognitive tests that MoCA-Impaired patients performed significantly poorer than MoCA-Intact patients and it can be classed a test of executive function. If we examine naming ability, almost all patients obtained full marks for the MoCA naming items, although $17.4 \%$ of all patients were impaired on the standard Graded Naming Test. Very few patients obtained full marks on the MoCA-specified memory items although, as noted above, a main limitation of the MoCA is that visual memory is not assessed.

The inclusion of all types of brain tumors in our study could be argued to limit our findings. This is unlikely for two reasons. First, in our study, patients with both meningiomas and gliomas (high/low-grade) were in the MoCA-impaired group (see Table 1). Secondly, in a recent study specifically investigating the effect of etiology on cognitive performance in patients with focal frontal lesion, once age and pre-morbid intelligence were accounted for, 
there were no significant differences between patients of different etiologies (stroke, meningioma, high/low-grade gliomas (42). One caveat, however, is the practical implications of treatments for different brain tumor types. For example, the timing of a brief CA in patients with higher grade gliomas who proceed to receive initial radiation or chemotherapy at 2-6 weeks post-resection, followed by a gap with no treatment and then adjuvant chemotherapy (43), needs to be considered in specific contexts. If a neuropsychologist is attached to an acute neurosurgical ward, then assessment prior to treatment can be included in routine planned care. If this is unavailable, then an optimal time would be in the gap between treatments, which would be approximately 8-10 weeks post-resection. Findings from a brief CA at either of these time points will be useful in further management, informing specific cognitive strategies/interventions and for the patient to understand changes in thinking related to their tumor.

In summary, the MoCA has extremely poor sensitivity to cognitive impairment in our primary brain tumor sample, which means that if a "normal" MoCA score is obtained, a CA is necessary. Even if a patient is impaired on the MoCA, the severity may be underestimated and some areas of cognition are not assessed. In fact, only one MoCA-specified domain showed even remotely similar detection levels as a brief CA. A full discussion of other brief $\operatorname{cog}$ nitive screening tools (e.g., ACE-III; CogMed) is beyond the scope of this preliminary study although we can speculate that similar issues would be revealed. Thus, despite the limitations of our small sample size, we strongly demonstrate that a brief and tailored CA lasting only $1-1.5 \mathrm{~h}$ is necessary and possible for the detection of cognitive impairments in primary brain tumor patients in the acute phase post-surgery. This is not only important for prognosis and monitoring, but it is crucial for neurorehabilitation and interventions $(1,2,4)$. Moreover, mental deterioration, or fear of this, was rated as one of the highest concerns of patients and carers, contributing to quality of life (20). Our study suggests that the critical cognitive domains to assess are executive functions (initiation, suppression, abstraction), attention, memory (verbal and visual), and language (naming and verbal fluency). Finally, we highly recommend adopting the neuropsychological principle of tailoring an assessment based on lesion location and presenting symptoms.

\section{ACKNOWLEDGMENTS}

We thank the patients for their participation. This research was supported by the NEWRO Foundation (Australia) and GR is supported by Australian Research Council Discovery Early Career Researcher Award (DE120101119).

\section{REFERENCES}

1. Meyers CA, Hess KR, Yung WK, Levin VA. Cognitive function as a predictor of survival in patients with recurrent malignant glioma. J Clin Oncol (2000) 18(3):646-50.

2. Taphoorn MJB, Klein D. Cognitive deficits in adult patients with brain tumours. Lancet Neurol (2004) 3:159-68. doi:10.1016/S1474-4422(04)00680-5

3. Olsen R, Iverson G, Carolan H, Parkinson M, Brooks B, McKenzie M. Prospective comparison of two cognitive screening tests; diagnostic accuracy and correlation with community integration and quality of life. J Neurooncol (2011) 105:337-44. doi:10.1007/s11060-011-0595-4
4. Papagno C, Casarotti A, Comi A, Gallucci M, Riva M, Bello L. Measuring clinical outcomes in neuro-oncology. A battery to evaluate low-grade gliomas (LGG). J Neurooncol (2012) 108:269-75. doi:10.1007/s11060-012-0824-5

5. Anderson SW, Damasio AR, Tranel D. Neuropsychological impairments associated with lesions caused by tumour or stroke. Arch Neurol (1990) 47:397-405 doi:10.1001/archneur.1990.00530040039017

6. Shallice T, Mussoni A, D’Agostino S, Skrap M. Right posterior cortical functions in a tumour patient series. Cortex (2010) 46(9):1178-88. doi:10.1016/j.cortex. 2010.04.005

7. Giovagnoli AR. Investigation of cognitive impairments in people with brain tumors. J Neurooncol (2012) 108:277-83. doi:10.1007/s11060-012-0815-6

8. Folstein MF, Folstein SE, McHugh PR. "Mini-mental state". A practical method for grading the cognitive state of patients for the clinician. J Psychiatr Res (1975) 12:189-98. doi:10.1016/0022-3956(75)90026-6

9. Brown PD, Buckner JC, O’Fallon JR, Iturria NL, Brown CA, O’Neill BP, et al. Effects of radiotherapy on cognitive function in patients with low-grade glioma measured by the Folstein mini-mental state examination. J Clin Oncol (2003) 21:2519-24. doi:10.1200/JCO.2003.04.172

10. Tucha O, Smely C, Lange KW. Verbal and figural fluency in patients with mass lesions of the left or right frontal lobes. J Clin Exp Neuropsychol (1999) 21(2):229-36. doi:10.1076/jcen.21.2.229.928

11. Nasreddine ZS, Phillips NA, Bédirian V, Charbonneau S, Whitehead V, Collin I, et al. The Montreal cognitive assessment, MoCA: a brief screening tool for mild cognitive impairment. J Am Geriatr Soc (2005) 53:695-9. doi:10.1111/j.15325415.2005.53221.x

12. Olsen R, Parkinson M, McKenzie M. Selection bias introduced by neuropsychological assessments. Can J Neurol Sci (2010) 37:264-8. doi:10.1017/ S0317167100010039

13. Olsen R, Tyldesley S, Carolan H, Parkinson M, Chanabhai T, McKenzie M. Prospective comparison of the prognostic utility of the min-mental state exam and the Montreal cognitive assessment in patients with brain metastases. Support Care Cancer (2011) 19:1849-55. doi:10.1007/s00520-010-1028-1

14. Cumming TB, Bernhardt J, Linden T. The Montreal cognitive assessment: short cognitive evaluation in a large stroke trial. Stroke (2011) 42:2642-4. doi:10.1161/STROKEAHA.111.619486

15. Schweizer TA, Al-Khindi T, Macdonald RL. Mini-mental state examination versus Montreal cognitive assessment: rapid assessment tools or cognitive and functional outcome after aneurysmal subarachnoid haemorrhage. J Neurol Sci Turk (2012) 316(137-140):137-40. doi:10.1016/j.jns.2012.01.003

16. Zhao J, Tang H, Sun J, Wang B, Chen S, Fu Y. Analysis of cognitive dysfunction with silent cerebral infarction: a prospective study in Chinese patients. Metab Brain Dis (2012) 27:17-22. doi:10.1007/s11011-011-9275-5

17. Bernstein IH, Lacritz LH, Barlow CE, Weiner MF, DeFina LF. Psychometric evaluation of the Montreal cognitive assessment (MoCA) in three diverse samples. Clin Neuropsychol (2011) 25(1):119-26. doi:10.1080/13854046.2010. 533196

18. Chan E, Khan S, Oliver R, Gill SK, Werring DJ, Cipolotti L. Underestimation of cognitive impairments by the Montreal cognitive assessment (MoCA) in an acute stroke unit population. J Neurol Sci (2014) 343(1-2):176-9. doi:10.1016/j.jns.2014.05.005

19. Lezak MD, Howieson DB, Loring DW. Neuropsychological Assessment. 4th ed. Oxford: Oxford University Press (2004).

20. Janda M, Steginga S, Dunn J, Langbecker D, Walker D, Eakin E. Unmet supportive care needs and interest in services among patients with a brain tumour and their carers. Patient Educ Couns (2008) 71:251-8. doi:10.1016/j.pec.2008.01.020

21. Cipolotti L, Warrington EK. Neuropsychological assessment. J Neurol Neurosurg Psychiatry (1995) 58:655-66. doi:10.1136/jnnp.58.6.655

22. Robinson G, Cipolotti L, Walker DG, Biggs V, Bozzali M, Shallice T. Verbal suppression and strategy use: a role for the right lateral prefrontal cortex? Brain (2015). doi:10.1093/brain/awv003

23. Robinson G, Shallice T, Bozzali M, Cipolotti L. The differing roles of the frontal cortex in fluency tests. Brain (2012) 135:2202-14. doi:10.1093/brain/ aws 142

24. Nelson HE, Willison J. The National Adult Reading Test. 2nd ed. Windsor: The NFER-Nelson Publishing Co, Ltd (1991).

25. Raven J. Advanced Progressive Matrices, Set 1. Oxford: Oxford Psychologists Press (1976).

26. Delis DC, Kaplan E, Kramer J. Delis-Kaplan Executive Function System: Examiner's Manual. San Antonio, TX: The Psychological Corporation (2001). 
27. Murphy P, Shallice T, Robinson G, MacPherson SE, Turner M, Woollett K, et al. Impairments in proverb interpretation following focal frontal lobe lesions. Neuropsychologia (2013) 51(11):2075-86. doi:10.1016/j.neuropsychologia.2013. 06.029

28. Wechsler D. Wechsler Adult Intelligence Scale - Third Edition. San Antonio, TX: The Psychological Corporation (1997).

29. Robertson IH, Ward T, Ridgeway V, Nimmo-Smith I. The structure of normal human attention: the test of everyday attention. J Int Neuropsychol Soc (1996) 2(6):525-34. doi:10.1017/S1355617700001697

30. Warrington EK. Recognition Memory Test. Windsor: The NFER-Nelson Publishing Co Ltd (1984).

31. Warrington EK. The Camden Memory Tests. Hove: Psychology Press (1996).

32. Warrington EK, James M. The Visual Object and Space Perception Battery. Bury St Edmunds: Thames Valley Test Company (1991).

33. McKenna P, Warrington EK. Testing for nominal dysphasia. J Neurol Neurosurg Psychiatry (1980) 43:781-8. doi:10.1136/jnnp.43.9.781

34. Warrington EK, McKenna P, Orpwood L. Single word comprehension: a concrete and abstract word synonym test. Neuropsychol Rehabil (1998) 8(2):143-54. doi:10.1080/713755564

35. Benton AL. Differential behavioral effects in frontal lobe disease. Neuropsychologia (1968) 6(1):53-60. doi:10.1016/0028-3932(68)90038-9

36. Burgess P, Shallice T. The Hayling and Brixton Tests: Test Manual. Bury St Edmunds: Thames Valle Test Company (1997).

37. Snaith RP, Zigmond AS. Hospital Anxiety and Depression Scale. London: NferNelson Publishing Company Ltd (1994).

38. Marin RS, Biedrzycki RC, Firinciogullari S. Reliability and validity of the apathy evaluation scale. Psychiatry Res (1991) 38:143-62. doi:10.1016/0165-1781(91) 90040-V

39. Law B, Young B, Pinsker D, Robinson G. Propositional speech in unselected stroke: the effect of genre and external support. Neuropsychol Rehabil (2014). doi:10.1080/09602011.2014.937443
40. Hoops S, Nazem S, Siderowf AD, Duda JE, Xie SX, Stern MB, et al. Validity of the MoCA and MMSE in the detection of MCI and dementia in Parkinson disease. Neurology (2009) 73(21):1738-45. doi:10.1212/WNL.0b013e3181c34b47

41. Baird A, Walker DG, Biggs V, Robinson GA. Selective preservation of the beat in apperceptive amusia: a case study. Cortex (2014) 53:27-33. doi:10.1016/j.cortex. 2014.01.005

42. Cipolotti L, Healy C, Chan E, Bolsover F, Lecce F, White M, et al. The impact of different aetiologies on the cognitive performance of frontal patients. Neuropsychologia (2015) 68:21-30. doi:10.1016/j.neuropsychologia.2014.12.025

43. Stupp R, Mason WP, van den Bent MJ, Weller M, Fisher B, Taphorn MJB, et al. Radiotherapy plus concomitant and adjuvant temozolomide for glioblastoma. N Engl J Med (2005) 352:987-96. doi:10.1056/NEJMoa043330

Conflict of Interest Statement: The authors declare that the research was conducted in the absence of any commercial or financial relationships that could be construed as a potential conflict of interest.

Received: 25 November 2014; accepted: 25 February 2015; published online: 11 March 2015.

Citation: Robinson GA, Biggs V and Walker DG (2015) Cognitive screening in brain tumors: short but sensitive enough? Front. Oncol. 5:60. doi: 10.3389/fonc.2015.00060 This article was submitted to Neuro-Oncology, a section of the journal Frontiers in Oncology.

Copyright $($ C) 2015 Robinson, Biggs and Walker. This is an open-access article distributed under the terms of the Creative Commons Attribution License (CC BY). The use, distribution or reproduction in other forums is permitted, provided the original author(s) or licensor are credited and that the original publication in this journal is cited, in accordance with accepted academic practice. No use, distribution or reproduction is permitted which does not comply with these terms. 